Hot Topics in Physical Information (HoTPI-2013)

International Journal of Modern Physics: Conference Series Vol. 33 (2014) 1460354 (13 pages)

(C) The Author

DOI: $10.1142 / \mathrm{S} 2010194514603548$

\title{
Irreversible information loss: Fundamental notions and entropy costs
}

\author{
Neal G. Anderson \\ Department of Electrical and Computer Engineering \\ University of Massachusetts Amherst \\ Amherst, MA 01003-9292, USA \\ anderson@ecs.umass.edu
}

Published 17 September 2014

\begin{abstract}
Landauer's Principle (LP) associates an entropy increase with the irreversible loss of information from a physical system. Clear statement, unambiguous interpretation, and proper application of LP requires precise, mutually consistent, and sufficiently general definitions for a set of interlocking fundamental notions and quantities (entropy, information, irreversibility, erasure). In this work, we critically assess some common definitions and quantities used or implied in statements of LP, and reconsider their definition within an alternative "referential" approach to physical information theory that embodies an overtly relational conception of physical information. We prove an inequality on the entropic cost of irreversible information loss within this context, as well as "referential analogs" of LP and its more general restatement by Bennett. Advantages of the referential approach for establishing fundamental limits on the physical costs of irreversible information loss in communication and computing systems are discussed throughout.
\end{abstract}

Keywords: Landauer's Principle; information erasure; physics of information.

\section{Introduction: Interrogating Landauer's Principle}

A typical statement of the "entropic form" of Landauer's Principle ${ }^{1}$ (LP) goes something like this:

LP: Erasure of an amount $\Delta \mathcal{I}_{\text {er }}$ of information from a physical system unavoidably results in an entropy increase of

$$
\Delta S \geq k_{B} \ln (2) \Delta \mathcal{I}_{e r}
$$

where $k_{B}$ is Boltzmann's constant.

A well known generalization of LP due to Bennett ${ }^{2}$, hereafter referred to as the "Landauer-Bennett Principle" (LBP), addresses a broader class of operations that includes erasure as a special case:

This is an Open Access article published by World Scientific Publishing Company. It is distributed under the terms of the Creative Commons Attribution 3.0 (CC-BY) License. Further distribution of this work is permitted, provided the original work is properly cited. 
LBP: (A)ny logically irreversible manipulation of information, such as the erasure of a bit or the merging of two computation paths, must be accompanied by a corresponding entropy increase in non-information bearing degrees of freedom of the information processing apparatus or its environment.

These and other statements concerning the dissipative costs of information processing can be interpreted unambiguously only if the key notions and quantities are made precise. Of any such statement, we must ask: Information about what? Quantified how? Entropy of what? Quantified how? What, physically, constitutes erasure of information and "logically irreversible manipulation of information"? How are information- and non-information-bearing degrees of freedom to be properly distinguished? The relevant literature reflects a variety of views on these questions, although they often go without explicit answers. This hinders comparative assessment of various interpretations, resolution of controversies, and application to concrete scenarios.

In this work, we explore key aspects of information, entropy, and other fundamental notions as they function collectively in LP, LBP, and other propositions that concern irreversible information loss and its dissipative consequences. We begin in Sec. 2 by critically examining information erasure and LP through the prism of two fundamental questions:

Q1: What is the nature of information content in a physical system? Is it self referential, i.e. a property of the system state alone, or does it depend on the relationship between the system state and something external to the system (an external "referent")?

Q2: What counts as information erasure? Is it necessarily the complete and unconditional resetting of the system state to a "standard" reset state, commonly known in the literature as "Landauer erasure," or are there broader classes of state transformations that qualify?

We first take information erasure to be Landauer erasure, thus tentatively answering Q2, and consider the resulting options for answering Q1. We argue that the options strongly recommend an answer to Q1 that favors a referential view of information. Next, in Sec. 3, we carry the "referential" view into a more general consideration of irreversible information loss. Here we introduce an entropic decomposition, enabled by the referential conception of information, that offers an alternative to the "all or nothing" practice of separating the degrees-of-freedom of a system into those that bear information and those that do not. We argue that this decomposition also enables an objective physical interpretation of the distinction between "known data" and "random data," upholding such a distinction while de-anthropomorphizing it. Finally, in Sec. 4, we prove an inequality that functions as a "referential analog" of LBP for classical information in generally quantum mechanical systems. These 
considerations underlie the "referential" approach to irreversible information loss and its dissipative consequences that we have developed and applied elsewhere ${ }^{3,4,5,6}$.

\section{Landauer Erasure}

Before considering Q1, let us first say what we can about any appropriate measure $\mathcal{I}$ of information content under Landauer erasure. A self evident requirement would seem to be that $\mathcal{I}_{\text {reset }}=0$, where $\mathcal{I}_{\text {reset }}$ is the information content of the reset state in a Landauer erasure protocol, to ensure that the state reset completely drains the system of its information content. With the additional condition that information content is always nonnegative, which we take as a given here, information content is nonincreasing under all allowable state resets (so the amount of information erased is nondecreasing, i.e. $\Delta \mathcal{I}_{\text {er }}=\mathcal{I}_{\text {pre }}-\mathcal{I}_{\text {reset }} \geq 0$ where $\mathcal{I}_{\text {pre }}$ is the information content of the system state before reset), and the amount of information erased upon reset becomes equal to - and solely dependent upon - that held in the system before erasure $\left(\mathcal{I}_{\text {pre }}\right)$. With this, let us consider options for answering Q1.

\subsection{Landauer Erasure of Self Information}

We first answer Q1 by regarding information as self referential, so information content is a property of the system state alone $\left(\mathcal{I}=\mathcal{I}^{\mathcal{S}}\right)$, and consider the implications for Landauer erasure. A seemingly natural choice for $\mathcal{I}$ is the system's self entropy $\left(\mathcal{I}^{\mathcal{S}}=S^{\mathcal{S}}\right)$, given the mathematical similarities between informationtheoretic and physical entropy measures and Shannon's conceptual association of information content with uncertainty and self entropy ${ }^{7}$. Bounds consistent with LP follow for widely studied simple cases if the entropy change on the left-hand side of the Landauer inequality (1) is interpreted as the change in the entropy of the environment $\mathcal{E}\left(\Delta S=\Delta S^{\mathcal{E}}\right)$ and the reset state is a pure (zero entropy) state of the system $\mathcal{S}$. Perhaps the best known example is a simple two-state system functioning as a one bit memory that is erased by always resetting the system to a predetermined data state. The information content (read: self entropy) of the storage state and reset state are $\mathcal{I}_{\text {pre }}^{\mathcal{S}}=1$ bit and $\mathcal{I}_{\text {reset }}^{\mathcal{S}}=0$ bits, respectively, giving $\Delta \mathcal{I}_{\text {er }}=1$ bit and, by LP, an environmental entropy increase of $\Delta \tilde{S}^{\mathcal{E}} \geq-k_{B} \ln (2) \Delta S^{\mathcal{S}}$ with $\Delta S^{\mathcal{S}}=-1{ }^{\text {a }}$ For a thermal environment at temperature $T$, the "energetic form" $\Delta E \geq k_{B} T \ln (2) \Delta \mathcal{I}_{\text {er }}$ of LP then gives the oft quoted environmental heating of at least $k_{B} T \ln (2)$ per erased bit (taking $\Delta E=\Delta E^{\mathcal{E}}$ ).

General conclusions regarding erasure-by-reset cannot, however, be drawn from this canonical example, which is characterized by an entropy-reducing state reset and a zero-entropy reset state. It's easy to find erasure-by-reset scenarios that do not share these features. Consider, for example, a classical ideal gas in a cylinder with

\footnotetext{
a This is equivalent to the Second Law $\left(\Delta S_{\text {tot }}=\Delta S^{\mathcal{S}}+\Delta S^{\mathcal{E}} \geq 0\right)$, but with $\Delta S^{\mathcal{S}}$ and $\Delta S^{\mathcal{E}}$ expressed in information-theoretic and thermodynamic units, respectively. Entropies expressed in thermodynamic units are denoted here and throughout by a tilde: $\tilde{S}=k_{B} \ln (2) S$.
} 
a removable partition that is used as a one-bit memory, where the bit is stored by conditionally compressing gas so it occupies either the left or right half of the cylinder and holding it there by insertion of the partition. Erasure can be accomplished in this canonical "ideal gas memory" by a process that unconditionally leaves the gas confined to one pre-selected "standard" side of the cylinder: the partition is removed (so the gas expands freely), the gas is compressed to the standard side via insertion of a piston and application of work, the partition is reinserted to confine the gas to the standard side, and the piston is removed. This erasure process reduces the entropy of the gas when its volume is halved in the compression step, consistent with the nonnegativity of $\Delta \mathcal{I}_{e r}$, but does not leave the gas in a zero entropy state since the entropy is positive for an ideal gas at finite temperature in any finite volume. If information content is associated with self entropy, then $\mathcal{I}_{\text {reset }}=S_{\text {reset }}^{\mathcal{S}} \neq 0$ : The state reset has left the system in a state with nonvanishing information content (by the self entropy measure) even though all traces of the pre-reset system state have been eliminated and the "stored bit" has clearly been erased.

The above scenario provides just one example of a state reset that, by the self entropy measure, does not erase information, since this measure cannot do the work of enforcing the condition $\mathcal{I}_{\text {reset }}=S_{\text {reset }}^{\mathcal{S}}=0^{\mathrm{b}}$. A self-referential alternative is to associate information with the entropy of only selected information-bearing degreesof-freedom of $\mathcal{S}$, as in LPB, although this notion is limiting for reasons discussed in Sec. 3. It's hard to imagine other self-referential measures - measures defined solely on the system state - that would not suffer from similar inadequacies given the criteria they must satisfy: all possible reset states must share the property $\mathcal{I}_{\text {reset }}=0$, all information-bearing states must share the property $\mathcal{I}_{\text {pre }}>0$, and $\mathcal{I}$ must depend on the system state alone. An additional problem for such measures is that they are inherently unable to distinguish erasure- $b y$-reset from erasure- $a s$-reset - i.e. to distinguish state reset processes that erase information from those that do not - which is a distinction we should reasonably expect to make in a physical theory of information. The assertion that information is erased every time the state of any physical system is reset to a standard state immediately begs the question "Information about what?," which is a question that self referential measures can answer only in a trivial sense.

\subsection{Landauer Erasure of Referential Information}

Next, we consider erasure-by-resetting with an alternative answer to Q1, by explicitly adopting a relational conception of information for which the information content $\mathcal{I}$ depends on the relationship between the state of a system $\mathcal{S}$ and that of an external referent $\mathcal{R}\left(\mathcal{I}=\mathcal{I}^{\mathcal{R} S}\right)$. As argued elsewhere ${ }^{3,4,5,6}$, an appropriate

\footnotetext{
bA loophole that would "save" this measure is the requirement that all reset states of the system have zero entropy, but this is clearly too restrictive. Zero-entropy states are hard to come by in reality, and scenarios are easily identified where information is erased by resetting systems to states with nonzero entropy (e.g. erasure of an ordinary computer memory via a CLEAR operation).
} 
measure is the (generally quantum) mutual information

$$
\mathcal{I}^{\mathcal{R S}}=S^{\mathcal{R}}+S^{\mathcal{S}}-S^{\mathcal{R} \mathcal{S}}
$$

"between" the system state and referent state, which is defined on the joint statistical state of $\mathcal{R S}$ and quantifies correlations between the states of the two systems. Several advantages of such a measure are immediately evident:

(1) The referential conception of information has a built-in answer to the question of what the information allegedly "contained" in the state of $\mathcal{S}$ is about: it is about the state of $\mathcal{R}$. This is the kind of information that is of interest in communication and computing systems. ${ }^{\mathrm{c}}$

(2) $\mathcal{I}^{\mathcal{R} S}$ enables a clear distinction between system states that hold all of the information about the state of $\mathcal{R}\left(\mathcal{I}^{\mathcal{R S}}=S^{\mathcal{R}}\right)$ and those that hold no information about the state of $\mathcal{R}\left(\mathcal{I}^{\mathcal{R S}}=0\right)$. It also allows quantification of how much information about $\mathcal{R}$ is held in $\mathcal{S}$ for intermediate cases, where $\mathcal{R}$ holds incomplete information about $\mathcal{S}$.

(3) Any protocol that unconditionally resets all possible pre-reset states of the system to a standard reset state has the property $\mathcal{I}_{\text {reset }}^{\mathcal{R S}}=0$, ensuring that

$$
\Delta \mathcal{I}_{\text {er }}=\mathcal{I}_{\text {pre }}^{\mathcal{R S}}-\mathcal{I}_{\text {reset }}^{\mathcal{R S}}>0
$$

for all information-bearing pre-reset states $\left(\mathcal{I}_{\text {pre }}^{\mathcal{R}}>0\right)$. This holds whether the system's self entropy is increased, decreased, or unchanged as a result of the state reset.

(4) $\mathcal{I}^{\mathcal{R S}}$ is nonincreasing for any local quantum operation on $\mathcal{S}^{\mathrm{d}}$ - unitary or nonunitary - that transforms the state of $\mathcal{S}$ while leaving the state of $\mathcal{R}$ unchanged.

Furthermore, as we have shown in previous work ${ }^{3}$, loss of referential information lower bounds the total von Neumann entropy increase $\left(\Delta S_{t o t}=S^{\mathcal{R} \mathcal{S}}+S^{\mathcal{E}}\right)$ as per the Landauer-like inequality

$$
\Delta \tilde{S}_{t o t} \geq-k_{B} \ln (2) \Delta \mathcal{I}^{\mathcal{R S}}
$$

This bound is obtained from quantum dynamics and entropic inequalities alone, and holds for any local operation performed unconditionally on $\mathcal{S}$ in interaction with $\mathcal{E}$. For the special case of a Landauer erasure operation - i.e. when $-\Delta \mathcal{I}^{\mathcal{R S}}=$ $\mathcal{I}_{\text {pre }}^{\mathcal{R}}=\Delta \mathcal{I}_{\text {er }}$ - we recover "referential forms" of Landauer's inequality and LP:

\footnotetext{
${ }^{\mathrm{c}}$ These systems are, of course, valued for their ability to transfer or process information about something external to themselves, something other than the randomness that results from interaction of the system with an inaccessible and uncontrollable environment. This "selective aboutness" should be naturally reflected in the concepts, definitions and quantitative measures employed in a fundamental physical description of computing and communication.

dAny evolution that transforms the state of $\mathcal{S}$ through work and environmental interactions is a local quantum operation on $\mathcal{S}$.
} 
$\mathbf{L P}(\mathbf{R})$ : Erasure of an amount $\Delta \mathcal{I}_{\text {er }}$ of information from a physical system $\mathcal{S}$ unavoidably results in a total entropy increase of

$$
\Delta \tilde{S}_{t o t} \geq k_{B} \ln (2) \Delta \mathcal{I}_{\text {er }}
$$

where $\Delta \mathcal{I}_{\text {er }}=\mathcal{I}_{\text {pre }}^{\mathcal{R S}}$ is the amount of information about a referent $\mathcal{R}$ that is erased from $\mathcal{S}$ and $k_{B}$ is Boltzmann's constant.

The inequality (5) holds for arbitrary changes in the self entropy of $\mathcal{S}$, with the condition $\mathcal{I}_{\text {reset }}^{\mathcal{R S}}=0$ automatically satisfied for all state reset operations. State resets that erase information $\left(\Delta \mathcal{I}_{e r}>0\right)$ are distinguished from state resets that do not $\left(\Delta \mathcal{I}_{\text {er }}=0\right)$, even when transformation of the local state of $\mathcal{S}$ is identical in the two cases. The key is the mutual information measure $\mathcal{I}^{\mathcal{R S}}$, which is decreasing under local operations on the system state that reduce its correlation with the referent, can never increase under such operations, and vanishes for all unconditional resets (which necessarily destroy all system-referent correlations).

Thus, by adopting a referential conception of information and the associated mutual information measure, conceptual and quantitative problems associated with consideration of Landauer erasure of self-referential information are alleviated. Beyond this, the referential approach naturally accommodates a more general consideration of irreversible information loss that includes partial erasure. Incorporation of partial erasure into a broader definition of erasure - an alternative answer to Q2 - seems appropriate in any case, as the strict, all-or-nothing notion of information erasure as state resetting is very limiting. Such a notion cannot make sense of scenarios in which imperfect state reset operations leave traces of the pre-reset state behind, or of physical implementations of logical operations that, by design, do not lose all input information. Landauer erasure is, in any case, of very limited applicability in the physics of computation, which should be concerned with a wide variety of practical and interesting information loss scenarios encountered in real computing devices. This motivates a broader view of information erasure processes - partial or complete - as special cases of irreversible information loss.

\section{Irreversible Information Loss}

The starting point for our more general reconsideration of irreversible information loss is the Landauer-Bennett Principle (see Sec. 1). This general statement about the entropy cost of information loss differs from Landauer's principle in two important respects. First, it accommodates partial information erasure (e.g. logically irreversible computation) as well as complete information erasure (e.g. via state resets). Second, the "information processing apparatus" has been conspicuously divided into information-bearing and non-information-bearing degrees of freedom.

As a stepping stone toward rigorous construction of a referential analog of LBP, we streamline and clarify this statement. We replace the phrase "logically irreversible manipulation of information" by "loss of information," remove the example 
processes provided in Bennett's statement, explicitly recognize that initially the "information" is necessarily held in information-bearing degrees of freedom of the system, and explicitly recognize that loss of information from the information-bearing degrees of freedom could generally increase the entropy of the non-informationbearing degrees of freedom and the environment (as opposed to "or the environment"). With these "friendly amendments," none of which are substantive, we can restate $\mathrm{LBP}$ as:

LBP': Any loss of information from the information-bearing degrees of freedom of an information processing apparatus must be accompanied by a corresponding net increase in the entropy of the non-information-bearing degrees of freedom of the apparatus and its environment.

To recast $\mathrm{LPB}^{\prime}$ in terms of a referential conception of information, we introduce a new entropic decomposition - enabled by the referential approach - that simplifies partitioning of the system's degrees of freedom into subsystems and allows the subsystem entropies to be decomposed in a new and useful way. The motivation for this will now be discussed.

\subsection{On Information-Bearing Degrees of Freedom}

Identification of the information-bearing degrees of freedom (IBDF) of a system, and thus "sorting" of a system's degrees of freedom (DF) into IBDF and non-IBDF (NIBDF), would seem to be straightforward enough: The IBDF are simply the degrees of freedom that, by whatever measure, hold the information! This sorting is straightforward provided that $(i)$ the IBDF can be unambiguously identified, $(i i)$ every DF holds either no information or nothing but information, and (iii) none of the IBDF (NIBDF) become "reclassified" as NIBDF (IBDF) as the system evolves in time. We now take a closer look at these criteria and reveal their limitations.

Regarding $(i)$, we do not deny that IBDF are easily identified and distinguished from NIBDF in simple, idealized systems - particularly macroscopic classical systems - where the information encoding strategy is clearly specified. This is, however, not the case in general. In engineered systems with limited controllability, such as complex nanoscale and quantum mechanical systems, information may become registered in "unintended" IBDF as well as the "intended" IBDF the engineer had in mind. In natural systems studied for their hypothesized information processing capacities, and in reservoir computing scenarios where the computing mechanisms are inherently "hidden," the IBDF are certainly not "given" a priori and may for all practical purposes be unknowable. If information is truly physical, then our general statements about relevant processes and their physical costs should be cast entirely in terms of physical states and dynamics without reference to isolation of IBDFs, since the identification and isolation of these degrees of freedom is generally uncertain even if it is assumed to be possible in principle.

Regarding ( $i i)$, we note that the "all-or-nothing" categorization of every degree 
of freedom as IBDF or NIBDF may not even be possible. There are no grounds for assuming that, in the general case, a system's degrees of freedom can always be partitioned into mutually exclusive sets of DF that hold only information and DF that hold no information. If this is not possible, so there exist some DF that hold some information but are also coupled to a random environment, should these DF be regarded as IBDF or a NIBDF? Is there an unambiguous, non-arbitrary way to make the assignment? Without evidence that a system's DF can always be meaningfully and unambiguously partitioned into a set of IBDF and a set of NIBDF, it is reasonable to question the usefulness, or at least the generality, of such a classification.

Finally, regarding (iii), we note that the identification of a degree of freedom as an IBDF or as NIBDF - if possible at all in light of the above considerations - is generally a time-dependent notion. Throughout a computational process, some DF that initially hold information may lose it completely and others that initially hold no information may gain some. This implies that DF classification is time dependent, which is problematic for several reasons. First, it is clearly at odds with the general practice of physical analysis, which begins with grouping degrees of freedom - once and for all - into fixed "subsystems" whose local states and observable properties vary with time but not their identities. Second, if DF can be "reclassified" during a physical process, it is hard to make sense of a statement like LBP. LBP specifies that the entropy of the NIBDF of an "information processing apparatus" may increase when information is lost from the IBDF of the apparatus, but is not explicit that this entropy increase generally involves a transfer of information to the NIBDF. This requires either that the NIBDF are reclassified as IBDF by virtue of having gained information, or that the NIBDF are not reclassified as IBDF and acquire the contradictory status of a non-information-bering entity that bears information. The interpretation of LPB, based as it is on decomposition of the information processing apparatus into IBDF and NIBDF, becomes ambiguous for these reasons and the others discussed above.

\subsection{From Coordinate Decomposition to Entropic Decomposition}

Using the referential conception of information, we can address the above concerns within a framework that allows decomposition of the "information processing apparatus" into fixed subsystems without requiring that they be categorized as IBDF or NIBDF. The apparatus degrees of freedom are partitioned and grouped - once and for all - into subsystems, and the entropy of each local subsystem state is decomposed into information-bearing entropy and non-information-bearing entropy. At any specified time, each subsystem holds an amount of information that is quantified by the information-bearing entropy of its state at that time.

To see how this entropic decomposition works, consider a system $\mathcal{S}$ in a state $\hat{\rho}^{\mathcal{S}}$ belonging to a composite quantum system $\mathcal{R S}$. The quantum mutual information 
$\mathcal{I}^{\mathcal{R S}}=S\left(\hat{\rho}^{\mathcal{R}}\right)+S\left(\hat{\rho}^{\mathcal{S}}\right)-S\left(\hat{\rho}^{\mathcal{R S}}\right)$ can be rearranged as

$$
S\left(\hat{\rho}^{\mathcal{S}}\right)=\mathcal{I}^{\mathcal{R S}}+\left\{S\left(\hat{\rho}^{\mathcal{R} \mathcal{S}}\right)-S\left(\hat{\rho}^{\mathcal{R}}\right)\right\} .
$$

Let $\mathcal{R S}$ be a classical-quantum referent-system pair, which is to say that $\hat{\rho}^{\mathcal{R} \mathcal{S}}$ is of the form

$$
\hat{\rho}^{\mathcal{R} \mathcal{S}}=\sum_{i} p_{i} \hat{\rho}_{i}^{\mathcal{R}} \otimes \hat{\rho}_{i}^{\mathcal{S}}
$$

where the $\hat{\rho}_{i}^{\mathcal{R}}=\left|x_{i}^{\mathcal{R}}\right\rangle\left\langle x_{i}^{\mathcal{R}}\right|$ are orthogonal (and thus distinguishable). Now look more closely at the two components of the system entropy $S\left(\rho^{\mathcal{S}}\right)$ :

- Evaluation of $\mathcal{I}^{\mathcal{R S}}$ for the referent-system pair yields

$$
\mathcal{I}^{\mathcal{R S}}=S\left(\rho^{\mathcal{S}}\right)-\sum_{i} p_{i} S\left(\rho_{i}^{\mathcal{S}}\right)
$$

which is immediately recognizable as the Holevo information $\chi\left(\epsilon^{\mathcal{S}}\right)$ defined for an ensemble $\epsilon^{\mathcal{S}}=\left\{p_{i}, \hat{\rho}_{i}^{\mathcal{S}}\right\}$ of quantum states. By Holevo's theorem ${ }^{8}, \chi\left(\epsilon^{\mathcal{S}}\right)$ is an upper bound on the accessible information $\mathcal{I}_{\text {acc }}$, which in this context is the amount of (classical) information about the state of $\mathcal{R}$ that can be obtained from the outcomes of an optimal measurement on $\mathcal{S}$. For mutually orthogonal $\rho_{i}^{\mathcal{S}}$, the upper bound $\chi\left(\epsilon^{\mathcal{S}}\right)$ is achievable with projective measurements, and is equal to the "preparation entropy" (i.e. the Shannon entropy $H\left(\left\{p_{i}\right\}\right)$ ). For nonorthogonal $\rho_{i}^{\mathcal{S}}$, the upper bound $\chi\left(\epsilon^{\mathcal{S}}\right)$ is achievable using a block coding scheme and joint measurments ${ }^{9,10}$ (but is less than $H\left(\left\{p_{i}\right\}\right)$ ). Taken together, these considerations strongly recommend identification of $\mathcal{I}^{\mathcal{R} \mathcal{S}}$ as the "informationbearing part" of the system entropy $S\left(\hat{\rho}^{\mathcal{S}}\right)$.

- Evaluation of $S\left(\hat{\rho}^{\mathcal{R S}}\right)-S\left(\hat{\rho}^{\mathcal{R}}\right)$ for the referent-system pair yields

$$
S\left(\hat{\rho}^{\mathcal{R S}}\right)-S\left(\hat{\rho}^{\mathcal{R}}\right)=\sum_{i} p_{i} S\left(\hat{\rho}_{i}^{\mathcal{S}}\right) \equiv\left\langle S_{i}^{\mathcal{S}}\right\rangle .
$$

This is simply the ensemble-averaged entropy of the individual preparations, i.e. the states $\hat{\rho}_{i}^{\mathcal{S}}$ in the ensemble $\epsilon^{\mathcal{S}}=\left\{p_{i}, \hat{\rho}_{i}^{\mathcal{S}}\right\}$. Note that, unlike $S\left(\hat{\rho}^{\mathcal{S}}\right),\left\langle S_{i}^{\mathcal{S}}\right\rangle$ is completely unrelated to the mutual distinguishability of the $\hat{\rho}_{i}^{\mathcal{S}}$ and their correlation with the states $\hat{\rho}_{i}^{\mathcal{R}}$ of the referent $\mathcal{R}$. It thus seems entirely reasonable to regard $\left\langle S_{i}^{\mathcal{S}}\right\rangle$ (and thus $S\left(\hat{\rho}^{\mathcal{R S}}\right)-S\left(\hat{\rho}^{\mathcal{R}}\right)$ ) as the "non-information-bearing part" of the system entropy.

With this, we can write the entropy of $\mathcal{S}$ as

$$
S\left(\hat{\rho}^{\mathcal{S}}\right)=\mathcal{I}^{\mathcal{R S}}+\left\langle S_{i}^{\mathcal{S}}\right\rangle
$$

where $\mathcal{I}^{\mathcal{R S}}$ is the information-bearing entropy and $\left\langle S_{i}^{\mathcal{S}}\right\rangle$ is the non-informationbearing entropy of $\mathcal{S}$. We emphasize that both components can be evaluated either from the joint state $(7)$ of $\mathcal{R S}$ or from the specification of the ensemble $\epsilon^{\mathcal{S}}=\left\{p_{i}, \hat{\rho}_{i}^{\mathcal{S}}\right\}$ 
of states of $\mathcal{S}^{\mathrm{e}}$. The sufficiency of $\hat{\rho}^{\mathcal{R} \mathcal{S}}$ is conceptually significant, as it qualifies the " $\mathcal{R}$-information in $\mathcal{S}$ " as a state quantity defined on the composite $\mathcal{R} \mathcal{S}$.

With this, we define irreversible loss of $\mathcal{R}$-information from $\mathcal{S}$ as a reduction in the information-bearing entropy $\mathcal{I}^{\mathcal{R} S}$ of $\mathcal{S}$. Any reduction in correlation between $\mathcal{R}$ and $\mathcal{S}$ necessarily results in creation of correlations between $\mathcal{R}$ and $\mathcal{E}$, which increases $S_{\text {tot }}$ and cannot be "reversed" by operations on $\mathcal{S}$ alone.

We suggest that this entropic decomposition, which enables unambiguous definition of irreversible information loss in the referential approach, also provides a natural, objective, and unambiguous distinction between "known data" and "random data." Association of "known data" with the information-bearing entropy $\mathcal{I}^{\mathcal{R} \mathcal{S}}$ - a quantity defined on the joint state of $\mathcal{R S}$ - provides a physically grounded definition of this quantity that addresses the obvious question "known by whom?" In the referential definition, this seemingly anthropomorphic question is replaced by "correlated to what?" The answer is, of course, the referent $\mathcal{R}$. If no such correlations exist $\left(\mathcal{I}^{\mathcal{R S}}=0\right)$, the case where $\mathcal{S}$ holds "random data," then all of the entropy $S\left(\hat{\rho}^{\mathcal{S}}\right)$ of $\mathcal{S}$ is necessarily non-information-bearing entropy ( $\left.c f .(10)\right)$. This is entirely appropriate since all of the randomness attributed to what has been called "random data" reflects interaction of the system $\mathcal{S}$ with an uncontrolled environment.

Having defined irreversible information loss within the referential approach, we note that the generic scenario used to motivate this definition leaves no room for reversible information loss. Because mutual information is conserved in a closed system, any $\mathcal{R}$-information lost from $\mathcal{S}$ is necessarily lost irreversibly to $\mathcal{E}$. To flesh out the distinction between irreversible and reversible information loss, and to quantify, compare and contrast their entropic costs, we must expand the description of the information processing apparatus $\mathcal{S}$. We do this below, and prove a theorem related to entropy and information loss within the referential approach that accommodates both irreversible and reversible information loss from $\mathcal{S}$ and makes use of the entropic decomposition outlined above. Our referential analog of $\mathrm{LBP}^{\prime}$ follows as a corollary.

\section{Entropy Cost of Information Loss in the Referential Approach}

We again consider a globally closed composite consisting of an information processing apparatus $\mathcal{S}$, an environment $\mathcal{E}$ with which $\mathcal{S}$ interacts, and a referent system $\mathcal{R}$, but introduce internal structure into the apparatus $\mathcal{S}$. Let the degrees of freedom of $\mathcal{S}$ be partitioned into two subsets. One subset is associated with an "information processing artifact" $\mathcal{A}$ - the part of the apparatus of particular interest for informational analysis - and the other (complementary) subset is associated with remainder $\overline{\mathcal{A}}$ of the apparatus. Either subsystem can hold partial or complete information about the state of the referent $\mathcal{R}$ at any time, but the partitioning is fixed.

e While the density operator $\hat{\rho}^{\mathcal{S}}$ follows directly from specification of an ensemble $\epsilon^{\mathcal{S}}$, the converse is not true: An infinite variety of ensembles are associated with a given density operator. 
The apparatus $\mathcal{A} \overline{\mathcal{A}}$ is distinguished from the environment $\mathcal{E}$ by the requirement that all controllable degrees of freedom belong to the apparatus and no controllable degrees of freedom belong to the environment.

Processing of $\mathcal{R}$-information by the apparatus requires unitary evolution of the composite $\mathcal{R} \mathcal{A} \overline{\mathcal{A}} \mathcal{E}$, consistent with Schrodinger dynamics and global closure of the composite. This is to say that any initial state $\hat{\rho}$ of $\mathcal{R} \mathcal{A} \overline{\mathcal{A}} \mathcal{E}$ evolves to a final state $\hat{\rho}^{\prime}=\hat{U} \hat{\rho} \hat{U}^{\dagger}$, where $\hat{U}=\hat{U}^{\mathcal{R} \mathcal{A} \overline{\mathcal{A}} \mathcal{E}}$ is the appropriate unitary evolution operator. If $\mathcal{R}$ is decoupled from $\mathcal{A} \overline{\mathcal{A}} \mathcal{E}$ throughout the operation, so the referent state remains intact and cannot influence the outcome of the operation, $\hat{U}$ is of the form $\hat{U}=\hat{U}^{\mathcal{R}} \otimes \hat{U}^{\mathcal{A} \mathcal{A} \mathcal{E}}$. With this, we state, prove, and discuss the following theorem:

Theorem 4.1. For unitary evolution

$$
\hat{\rho}^{\prime}=\left(\hat{U}^{\mathcal{R}} \otimes \hat{U}^{\mathcal{A} \overline{\mathcal{A}} \mathcal{E}}\right) \hat{\rho}\left(\hat{U}^{\mathcal{R}} \otimes \hat{U}^{\mathcal{A} \overline{\mathcal{A}} \mathcal{E}}\right)^{\dagger}
$$

of a closed composite $\mathcal{R} \mathcal{A} \overline{\mathcal{A}} \mathcal{E}$ in initial state

$$
\hat{\rho}=\left(\sum_{i} p_{i}\left(\hat{\rho}_{i}^{\mathcal{R}} \otimes \hat{\rho}_{i}^{\mathcal{A} \overline{\mathcal{A}}}\right)\right) \otimes \hat{\rho}^{\mathcal{E}}
$$

the entropy change $\Delta S^{\mathcal{E}}=S\left(\hat{\rho}^{\mathcal{E}^{\prime}}\right)-S\left(\hat{\rho}^{\mathcal{E}}\right)$ of the environment is lower bounded as

$$
\Delta S^{\mathcal{E}} \geq \Delta \mathcal{I}^{\mathcal{A} \overline{\mathcal{A}}}-\Delta \mathcal{I}^{\mathcal{R} \mathcal{A}}-\Delta\left\langle S_{i}^{\mathcal{A}}\right\rangle-\Delta S^{\overline{\mathcal{A}}}
$$

Proof. It follows from the form of (12) and the locally unitary evolution of $\mathcal{A} \overline{\mathcal{A}} \mathcal{E}$ that $\Delta S^{\mathcal{A} \overline{\mathcal{A}}}+\Delta S^{\mathcal{E}} \geq 0$, or

$$
\Delta S^{\mathcal{E}} \geq S\left(\hat{\rho}^{\mathcal{A} \overline{\mathcal{A}}}\right)-S\left(\hat{\rho}^{\mathcal{A} \overline{\mathcal{A}}^{\prime}}\right) .
$$

Writing $\mathcal{I}^{\mathcal{A} \overline{\mathcal{A}}}=S\left(\hat{\rho}^{\mathcal{A}}\right)+S\left(\hat{\rho}^{\overline{\mathcal{A}}^{\prime}}\right)-S\left(\hat{\rho}^{\mathcal{A} \overline{\mathcal{A}}^{\prime}}\right)$ (and similar for $\left.\mathcal{I}^{\mathcal{A} \overline{\mathcal{A}}^{\prime}}\right)$, we have

$$
\Delta S^{\mathcal{E}} \geq \Delta \mathcal{I}^{\mathcal{A} \overline{\mathcal{A}}}-\Delta S^{\mathcal{A}}-\Delta S^{\overline{\mathcal{A}}}
$$

The $\Delta S^{\mathcal{A}}$ term can be expressed as

$$
-\Delta S^{\mathcal{A}}=-\Delta \mathcal{I}^{\mathcal{R} \mathcal{A}}-\Delta\left\langle S_{i}^{\mathcal{A}}\right\rangle
$$

since, for the form (12),

$$
\begin{aligned}
\hat{\rho}^{\mathcal{R A}}=\operatorname{Tr}_{\overline{\mathcal{A}}}\left[\hat{\rho}^{\mathcal{R} \mathcal{A} \overline{\mathcal{A}}}\right] & =\sum_{i} p_{i} \hat{\rho}_{i}^{\mathcal{R}} \otimes \hat{\rho}_{i}^{\mathcal{A}} \\
\mathcal{I}^{\mathcal{R} \mathcal{A}} & =S\left(\hat{\rho}^{\mathcal{A}}\right)-\left\langle S_{i}^{\mathcal{A}}\right\rangle
\end{aligned}
$$

(and similar for $\hat{\rho}^{\mathcal{R} \mathcal{A}^{\prime}}$ and $\mathcal{I}^{\mathcal{R} \mathcal{A}^{\prime}}$ ). Substitution into (15) yields (13).

Now, the inequality (13) can be rearranged to give the key result of this work:

$$
\Delta\left\langle S_{i}^{\mathcal{A}}\right\rangle+\Delta S^{\overline{\mathcal{A}}}+\Delta S^{\mathcal{E}}-\Delta \mathcal{I}^{\mathcal{A} \overline{\mathcal{A}}} \geq-\Delta \mathcal{I}^{\mathcal{R} \mathcal{A}} .
$$

Put into words, this inequality says that 
"Any loss $-\Delta \mathcal{I}^{\mathcal{R} \mathcal{A}}$ of information from a subsystem $\mathcal{A}$ of an informationprocessing apparatus $\mathcal{A} \overline{\mathcal{A}}$ must be accompanied by a corresponding net increase in the non-information-bearing entropy $\left\langle S_{i}^{\mathcal{A}}\right\rangle$ of $\mathcal{A}$ and the total entropy $S^{\overline{\mathcal{A}}}+S^{\mathcal{E}}$ of the remainder of the apparatus $\overline{\mathcal{A}}$ and its environment $\mathcal{E}$, less the gain of intra-apparatus mutual information $\mathcal{I}^{\mathcal{A} \overline{\mathcal{A}}}$."

This very general statement clearly "parallels" LPB', the most conspicuous difference being the presence of the intra-apparatus mutual information change $\Delta \mathcal{I} \mathcal{A} \overline{\mathcal{A}}$ appearing in the bound (19). This term is very significant, as it accounts for the reduced entropy cost of information loss from $\mathcal{A}$ that becomes possible when $\mathcal{A}$ interacts with a preexisting copy of $\mathcal{R}$-information held in $\overline{\mathcal{A}}$. An example is the ERASE WITH COPY operation, in which the information-bearing state of $\mathcal{A}$ is reset in interaction with an exact copy that is held in $\overline{\mathcal{A}}$ throughout the erasure operation. This is a prominent example of reversible loss of information from $\mathcal{A}$; there is no irreversible loss of information from $\mathcal{S}$ to $\mathcal{E}$ in the ERASE WITH COPY operation, and the initial state of $\mathcal{A}$ can in principle be fully restored by local operations on $\mathcal{S}$ provided that the copy held in $\overline{\mathcal{A}}$ is preserved.

The intra-apparatus mutual information is not, however, part of LPB. To obtain our referential analog of $\mathrm{LBP}^{\prime}$, we consider the case where $\Delta \mathcal{I}^{\mathcal{R} \mathcal{A}}>0$ and $\Delta \mathcal{I}^{\mathcal{A} \overline{\mathcal{A}}}=$ 0 , so $\mathcal{R}$-information is initially held only in subsystem $\mathcal{A}$. Noting that $\mathcal{I}^{\mathcal{A} \overline{\mathcal{A}}}$ must be nonnegative at all times, so $\Delta \mathcal{I}^{\mathcal{A} \overline{\mathcal{A}}}=\mathcal{I}^{\mathcal{A} \overline{\mathcal{A}}^{\prime}} \geq 0$, the looser bound

$$
\Delta\left\langle S_{i}^{\mathcal{A}}\right\rangle+\Delta S^{\overline{\mathcal{A}}}+\Delta S^{\mathcal{E}} \geq-\Delta \mathcal{I}^{\mathcal{R} \mathcal{A}}
$$

follows as a corollary to Theorem 4.1. This is our referential analog of LPB':

$\mathbf{L B P}(\mathbf{R})$ : Any loss of information from a subsystem $\mathcal{A}$ of an informationprocessing apparatus must be accompanied by a corresponding net increase in the non-information-bearing entropy of $\mathcal{A}$ and the total entropy of the remainder of the apparatus and its environment.

All questions posed in Sec. 1 are answered for $\operatorname{LBP}^{\prime}(\mathrm{R})$ in the definitions of the quantities appearing in (20), as is the case for the more general inequality (19) and for $\operatorname{LP}(\mathrm{R})$ (5). We should note that no general claims are made here for the achievability of equality in these bounds, either in principle or in practice, simply because equality conditions have not been addressed in our proofs.

\section{Acknowledgments}

This work was supported in part by NSF Grant CCF-0916156. The author thanks Craig Lent for constructive criticism that sharpened several ideas presented here, and Ilke Ercan and Natesh Ganesh for insightful comments on the manuscript.

\section{References}

1. R. Landauer, IBM J. Res. Dev. 5, 183 (1961). 
2. C.H. Bennett, Studies in History and Philosophy of Modern Physics 34, 501 (2003).

3. N.G. Anderson, Physics Letters A 372, 5552 (2008).

4. N.G. Anderson, Theoretical Computer Science 411, 4179 (2010).

5. N.G. Anderson, Physics Letters A 376, 1426 (2012).

6. N. Ganesh and N.G. Anderson, Phys. Lett. A 377, 3266 (2013).

7. C.E. Shannon, Bell Syst. Tech. J. 27, 379 (1948).

8. A.S. Kholevo, Probl. Inf. Transm. 9, 177 (1973). A contemporary proof of an accessible information bound slightly tighter than $(\mathrm{K} / \mathrm{H})$ olevo's can be found in B. Schumacher, M. Westmoreland, and W.K. Wootters, Physical Review Letters 76, 3452 (1996).

9. A.S. Holevo, IEEE Transactions on Information Theory 44, 269 (1998).

10. B. Schumacher and M. Westmoreland, Physical Review A 56, 131 (1997). 